\title{
REAL-TIME MEASUREMENT OF DIELECTRIC RELAXATION OF BIOMOLECULES: KINETICS OF A PROTEIN-LIGAND BINDING REACTION*
}

\author{
Walter Scheider \\ Biophysics Research Division, Institute of Science and Technology \\ University of Michigan \\ Ann Arbor, Michigan 48109
}

\section{INTRODUCTION}

Some chemical reactions involving biomolecules are known to be accompanied by measurable changes in electric properties. The binding of long-chain fatty acids and similar ligands to human serum albumin (HSA) is one such reaction; a change in the dipole vector of the protein has been documented.'

We have used this property to develop a new probe to study the kinetics of these binding reactions.

The probe consists of a measurement of the dielectric properties of a reacting mixture, made by recording the response in real time to a single electric pulse. This technique has the advantage of providing several parameters from each measurement, which, in the experiments we are reporting here, provided us with simultaneous access to two separable measures of reaction progress. This resulted in identification of two distinct steps in the binding reaction.

The observations are made on rapidly mixed solutions of HSA and ligand, with use of stop-flow methods. Each observation requires the recording of data for an interval of $20-50 \mu \mathrm{sec}$, and is repeated at intervals from $1 \mathrm{msec}$ to $20 \mathrm{sec}$ after mixing.

\section{Single-Pulse Dielectric Relaxation Measurement}

Ever since Debye's classical explanation ${ }^{2}$ of the mechanism by which polar molecules orient in an electric field and the subsequent discoveries in the $1940 \mathrm{~s}^{3,4}$ that many protein molecules in solution behave in an electric field very nearly like polar molecules of an ideal gas, it has been only a matter of time and technology before the orientation of biologically important molecules could be observed as it happens - in real time.

By a Fourier transformation one can go from a description of a system response in the frequency domain to a description in the time domain, and, as Dr. Wada has pointed out (Husimi and $\mathrm{Wada}^{5}$ ) in the context of his very excellent method of applying a burst of pseudorandom noise, any transient signal that contains as Fourier components the relevant parts of the frequency spectrum, will, in principle, yield the same information as the traditional frequency scan.

Of course the business of finely tuning a test circuit one frequency at a time provides an enormous, useful redundancy in the desired information, allowing one

*Supported by the Institute of General Medical Sciences, NIH grant GM 22309. 
to amplify the wanted signal greatly while eliminating almost all nonsystematic error.

One reduces this desirable redundancy when one reduces the number of times one repeats the essential features of the test wave shape; one loses in signal-tonoise ratio, and consequently in accuracy, as one shortens the measurement time. Dr. Wada's method, which he will be discussing later, strikes an excellent balance between speed and accuracy.

The method we have developed consists of applying a single square pulse of the order of $20-50 \mu \mathrm{sec}$ duration, essentially limiting the measurement period to the response time of the sample. In so doing we sacrifice further the ability to filter out noise, but, as it turns out, the information yield is still quite satisfactory for a number of purposes.

We expect the usefulness of the single-pulse method to be primarily in three categories of experiments: 1) those in which it is difficult to maintain the physical environment constant for steady state measurements; 2) those where intentional changes in the sample are induced, and repeated single-pulse measurements are made to observe the resultant sample changes; and 3) those where the pulse itself may cause sample "damage" and one has essentially only one shot at making a measurement. Our present use of the method is in a system of category 2).

\section{Design, Theory, Data Processing;}

The pulse method involves first of all a choice of pulse shape. The square pulse, or Heaviside function, has a spectral density inversely proportional to the fre-

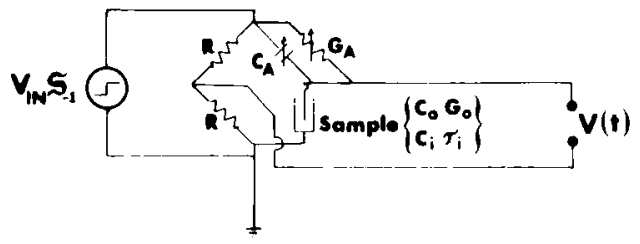

Figure I. Schematic of bridge arrangement for single-pulse response measurement of diefectric relaxation of a sample placed between electrodes (see text for definition of symbols).

quency, and thus is uniform on a $\log$ frequency scale. This corresponds most closely to the customary and most efficient sampling density used in frequency scans for obtaining Debye dispersion curves, and so it becomes the pulse shape of choice.

One seeks next to maximize the proportion of response signal that reveals desired information, and one does this by balancing out what might be called the base-line capacitance and conductance of the sample in a bridge arrangement.

For purposes of analysis, the sample is specified in terms of its conductance, $G_{0}$; its frequency-independent capacitance, $C_{0}$; and dispersion components " $i$ " of increment $C_{i}$ and relaxation time, $\tau_{i}$. One aims to balance out $C_{0}$ and $G_{0}$ by setting the balance arm capacitance and conductance to estimates of these values, $C_{A}$ and $G_{A}$. Fixed and equal low resistances $R$ make up the other two bridge arms, as in Figure 1 . In this arrangement, the input signal is a step function ${ }_{-1}$ (using the notation of Ref.6) of amplitude $V_{I N}$. 
The circuit of Figurs I may be solved in the frequency domain. The response $V(t)$ in the time domain to a signal $V_{\mathrm{IN}}$ is then obtained by multiplying the frequency response by the spectral density $(j \omega)^{-1}$, where $j$ is the unit imaginary and $\omega$ the angular frequency, and obtaining the Fourier transform of the resultant quantity. For the case of a single relaxation component $\left(C_{1}, \tau_{1}\right)$, one obtains

$$
\begin{aligned}
& \frac{V(t)}{V_{\mathrm{IN}}}=\frac{1}{2} \frac{\left(G_{A}-G_{0}\right)}{\left(G_{A}+G_{0}\right)}+\frac{1}{\tau_{1}\left(C_{A}+C_{0}\right)\left(\beta_{1}-\beta_{2}\right)} \sum_{i=1}^{2} \\
& (-1)^{i} e^{-\beta_{i}{ }^{\prime}\left(1-\beta_{i} \tau_{1}\right)\left(C_{A}-\frac{G_{A}}{\beta_{i}}\right)}
\end{aligned}
$$

where $\beta_{1}$ and $\beta_{2}$ are the roots of the equation

$$
\tau_{1} \beta^{2}-B \beta+\tau_{0}^{-1}=0
$$

and

$$
\begin{gathered}
\tau_{0}=\frac{C_{A}+C_{0}}{G_{A}+G_{0}} \\
B=1+\frac{C_{1}}{C_{A}+C_{0}}+\frac{\tau_{1}}{\tau_{0}}
\end{gathered}
$$

$\tau_{0}$ can be thought of as a relaxation time of the fixed quantities alone.

For two components of increment $C_{1}$ and $C_{2}$ and time constants $\tau_{1}$ and $\tau_{2}$, one obtains

$$
\begin{aligned}
\frac{V(t)}{V_{\mathrm{IN}}}=\frac{1}{W\left(C_{A}+C_{0}\right)} \prod_{i=1}^{3} \gamma_{i}^{-1} \sum_{i=1}^{3} \gamma_{i}\left\{\frac{-G_{A}}{\beta_{i}}+\left[\frac{G_{A}}{\beta_{i}}\right.\right. \\
\left.\left.-\left(C_{A}+G_{A} T\right)+\beta_{i}\left(W G_{A}+C_{A} T\right)-\beta_{i}^{2} C_{A} W\right] e^{-\beta_{i}}\right\}-\frac{1}{2}
\end{aligned}
$$

where $\beta_{i}$ are the roots of

$$
W \beta^{3}-Q \beta^{2}+R \beta-\tau_{0}^{-1}=0
$$

and

$$
\begin{aligned}
\gamma_{1} & =\beta_{2}-\beta_{3}: \gamma_{2}=\beta_{3}-\beta_{1}: \gamma_{3}=\beta_{1}-\beta_{2}: \\
T & =\tau_{1}+\tau_{2} \\
W & =\tau_{1} \tau_{2} \\
R & =1+T / \tau_{0}+\left(C_{1}+C_{2}\right) /\left(C_{A}+C_{0}\right) \\
Q & =T+W / \tau_{0}+\left(C_{1} \tau_{2}+C_{2} \tau_{1}\right) /\left(C_{A}+C_{0}\right)
\end{aligned}
$$

and $\tau_{0}$ is defined as in $(1)$.

Some simple response characteristics can be described. The value of $V(0)$, at time $t=0$, is always determined by the balance of $C_{A}$ and $C_{0}$, and if these 


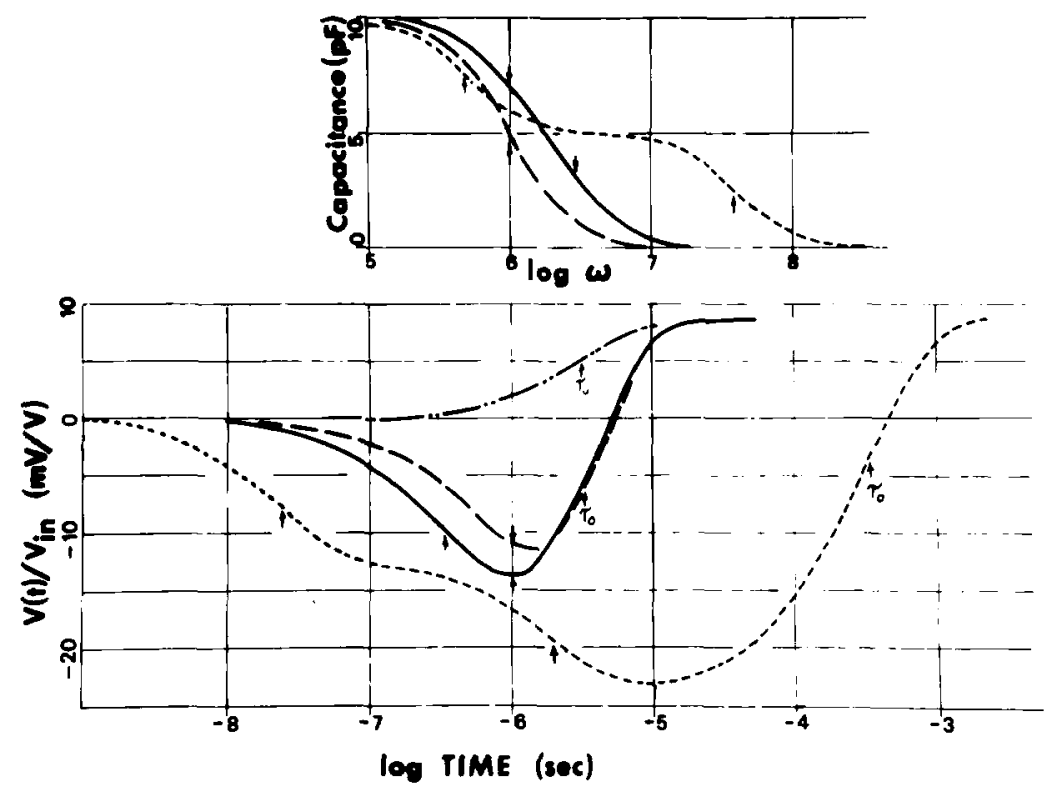

Figlar 2. Calculated response curves in the lime domain and (above) corresponding Debye dispersion curves in the frequency domain for the same parameters. Time domain data calculated from Equation 2: $C_{A}=C_{0}=100 \mathrm{pF}$ for all cases. $-\cdots=$ no relaxation components, $\tau_{0}=3.4 \mu \mathrm{sec}$ : $\ldots . . .-$ widely separated dispersion regions, $C_{1}=$ $C_{2}=5 \mathrm{pF}, \tau_{1}=.025 \mu \mathrm{sec}, \tau_{2}=2 \mu \mathrm{sec}, \tau_{0}=340 \mu \mathrm{sec}:-$ simulated data for globular proteins, $C_{1}=C_{2}=5 \mathrm{pF}, \tau_{1}=.333 \mu \mathrm{sec}, \tau_{2}=1 \mu \mathrm{sec}, \tau_{0}=3.4 \mu \mathrm{sec} ;$ and $-=$ single relaxation time, $C_{1}=10 \mathrm{pF}, \tau_{1}=1 \mu \mathrm{sec}, \tau_{0}=3.4 \mu \mathrm{sec}$. Arrows indicate relaxation times or relaxation frequencies.

are equal, the response always begins at the origin (FIGURE 2). At large times, $V(t \rightarrow x)$ approaches the asymptotic values $1 / 2\left(G_{A}-G_{0}\right) /\left(G_{A}+G_{0}\right)$. If there is no dispersion, it approaches this asymptote exponentially with the time constant $\tau_{0}$ (dash - dot curve).

If there is one dispersion region, and $\tau_{1} \ll \tau_{0}$, one observes first the polarization due to molecular orientation, the effect of which is to increase the sample capacitance with time. This is followed by the asymptotic approach to $V(x)$.

Figure 2 contains three simulated-response curves in both time and frequency domains for systems with dispersion. The dotted line represents the case in which $\tau_{1}, \tau_{2}$ (arrows) and $\tau_{0}$ are widely separated; this response function is a sum of three exponential decay terms (2), and thus the separation of the relaxation domains is qualitatively different from the corresponding separation obtained in the frequency domain, where the response is a sum of Lorentzian terms. The other two response curves shown correspond more nearly to the dispersion properties of globular proteins, where $\tau_{1}$ and $\tau_{2}$ span at most one decade, and where the solution conductance at best gives a value of $\tau_{0}$ somewhat larger than the molecular relaxation times. 
FIGURE 3(a) shows experimental time domain response data from a sample of HSA. Such data can be fitted by a one- or two-relaxation time representation directly, using Equations 1 and 2, respectively.

If one is interested in fitting such data with a more complicated model, involving more than two relaxation times, or a continuous distribution of relaxation times as in Cole-Cole equation,

$$
\epsilon(\omega)=\epsilon_{s}+\frac{\epsilon_{1}}{1+(j \omega \tau)^{\alpha}}
$$

the lack of linear additivity of relaxation components in the time-domain response functions poses great computational difficulties. In Equation 3, $\epsilon$ is the dielectric constant, $\epsilon_{s}$ the solvent dielectric constant, $\epsilon_{1}$ the dielectric increment, and $\alpha$ is a number between 0 and 1 .

Fitting to such more complicated models can be done by computing a Fourier Transform ${ }^{8}$ of the time-domain data, and obtaining from that, by straight forward algebraic manipulation, a conventional Debye representation of dielectric increment in the frequency domain, of which the real part is shown in Figure 3(b). The transformed data can then be fitted in the frequency domain where relaxation components add linearly. The two principal drawbacks to this method are 1) the ability to assign appropriate weighting factors to the various portions of data is largely lost, and 2) the transformation almost always requires extensive interpolation and extrapolation of the actual data, which is difficult to do without biasing the information.

The electronics used in making these measurements were custom-designed

Figure 3. (a): Data taken on a sample of Human Serum Albumin, $41.2 \mathrm{~g} / \mathrm{l}$ in water, Run $26: 3$, in a cell with cell constant $11.52 \mathrm{~cm}$., at $0^{\circ} \mathrm{C}$; (b): Debye dispersion curve from same data calculated by means of a Fourier Transformation.
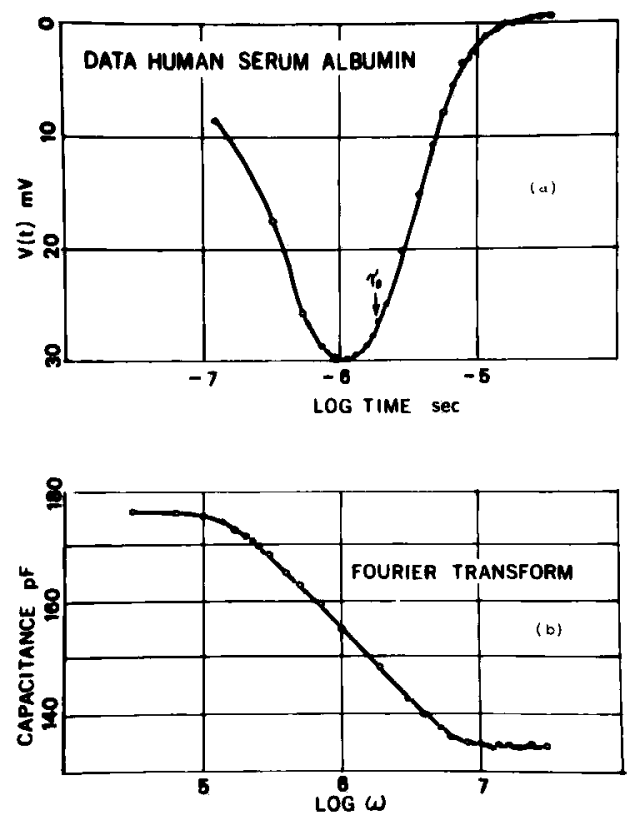
to permit programming of pulse width, repetition rate, and data-sampling times. The bridge output is amplified and sampled at intervals of $200 \mathrm{nsec}$, converted to digital form with 8-bit accuracy, and stored in a memory for later retrieval and processing.

\section{ObSERVING Ion ImMobilization and Binding IN REAL-Time}

Our initial interest in the ability to make these extremely fast measurements of dielectric relaxation was in its application to kinetic studies of the reactions between human serum albumin and small molecules.

The steady state dielectric properties of serum albumin solutions have been well characterized, ${ }^{4,9-11}$ and in particular it is known that the dielectric increment is markedly altered by the binding of fatty acids.'

The equilibrium constants of such binding have been studied by chemical methods ${ }^{12,13}$ and, for the first several moles of fatty acid bound, are of the order of $10^{7} \mathrm{M}^{-1}$. This extremely tight binding accounts for the difficulty in completely defatting this protein. ${ }^{14,15}$ These and other observations ${ }^{16-18}$ have supported the view that two or three of the primary sites for fatty acid and certain other organic ligands are in a portion of the albumin surface which is interior to the molecule in its normal, or $N$, form. Studies of the $N-F$ transition, induced by lowering the $\mathrm{pH}$ to approximately 4 , are consistent with the hypothesis that this interior surface becomes more accessible to the solvent in the $F$-form, accounting for the relative ease with which in vitro defatting occurs at lower $\mathrm{pH}$ values.

In our current research we use the electric pulse as a probe of the changes occurring in the albumin molecule as free fatty acid is suddenly made available for binding. This condition is created in a stop-flow apparatus, which is conventional in design, ${ }^{19}$ except that after the albumin solution and the solution of fatty acid come together, the mixture is observed in an electrode chamber instead of in the customary optical cell.

Our mixing cell with electrode chamber (Figure 4) has a mixing time of $<2$ msec., mixing volume $=40 \mu \mathrm{l}$, electrode chamber volume $=14 \mu \mathrm{l}$, and a cell constant $=14.8 \mathrm{~cm}$.

Our HSA was obtained as a reworked fraction $\mathrm{V}^{20}$ from the Blood Fractionation Laboratory of the Michigan Department of Public Health, Lansing, Michigan. It contained a maximum of $3^{\circ}$ " nonalbumin proteins, and less than $5^{\circ}$ "of dimer, as characterized both by ultracentrifugation and by gel filtration.

The protein is first defatted by the method of Ref. 21 , using XAD-2 resin, and a speeded-up technique for bringing the protein to defatting $\mathrm{pH}$ and returning it to $\mathrm{pH} 5$ immediately on exit from the resin column. The defatted protein is found to be labile, and to denature if left at low $\mathrm{pH}$ longer than several hours.

Numerous technical problems are associated with the introduction of longchain fatty acids into the protein solution, related to the fact that these ligands are micelle-formers in water. Consequently also the assignment of such quantities as the free ligand concentration is not straightforward.

We have obtained results from stop-flow mixtures of albumin solutions with 1) dispersions of oleic acid in $10 \%$ Ethanol-water mixtures, 2) solutions of sodium oleate, and 3 ) solutions of the more soluble ligand, $N$-acetyl tryptophan. Sim- 
ilarities in the binding of fatty acids and of $N$-acetyl tryptophan were reported by Dintzis. $^{22}$

In each experiment, one obtains a time-domain response curve such as is shown in FIGURE 3(a) immediately upon stopping of the mixture of HSA and ligand solution in the electrode chamber; one then repeats the application of a pulse and recording of a response curve in a digital memory, at various intervals during the course of the chemical reaction; i.e., the binding of the ligand.

From the series of response curves we routinely calculate values for the parameters $G_{0}, C_{0}, C_{1}, \tau_{1}, C_{2}, \tau_{2}$, and we can also obtain by Fourier Transformation a Cole-Cole parameter $\alpha$ in the $\tau_{1}$ component of increment. However, $C_{0}, \tau_{1}$, and $\tau_{2}$ are assumed not to be parameters of the reaction, and $C_{1}$ and $C_{2}$ are not even under the best circumstances ${ }^{1}$ determinable separately with great certainty.

As reaction-sensitive parameters, we thus obtain the total increment $C_{\text {tot }}=$

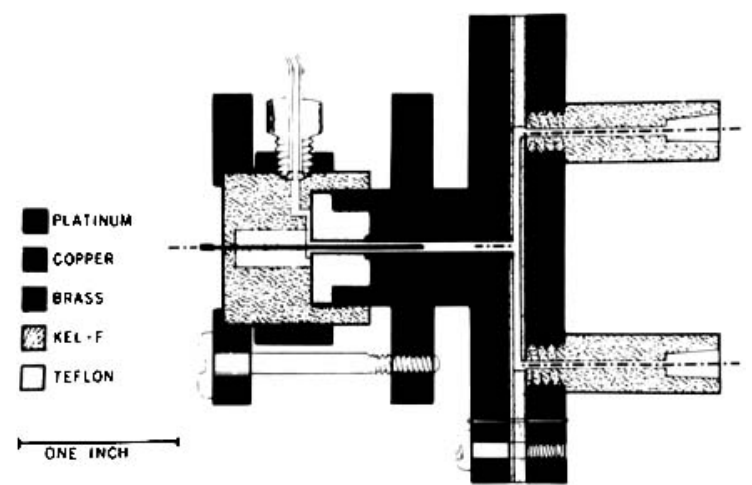

Figure 4. Schematic cross section of stop-flow dielectric cell. Reactants enter at the two Luer fittings at the right, mix in a complex $T$ mixer in the Teflon" sandwich (details of mixer configuration not shown), and pass to the platinum electrode chamber.

$C_{1}+C_{2}$, and $G_{0}$; these, in fact, provide us with the ability to monitor simultaneously two different events involved in the reaction.

$G_{0}$, the solution conductance, changes as an ionic species becomes relatively immobilized by binding to a large molecule.

$C_{\text {tot }}$, the total increment of capacitance due to molecule orientation, gives an indication of the extent to which the reaction has proceeded to its final equilibrium state.'

In FIGURE 5 are shown the values of $C_{\text {tot }}$ as a function of time after stop-flow mixing, calculated from a sequence of pulse-response curves obtained during the reaction of HSA with a solution of equal molarity of $N$-acetyl-DL-tryptophan. Also shown are results from a control mixture of HSA and water. There are several possible explanations of the data from $1 \mathrm{sec}$ to $20 \mathrm{sec}$, which will be elucidated in further experiments. In this report we have analyzed the data up to $1 \mathrm{sec}$ 


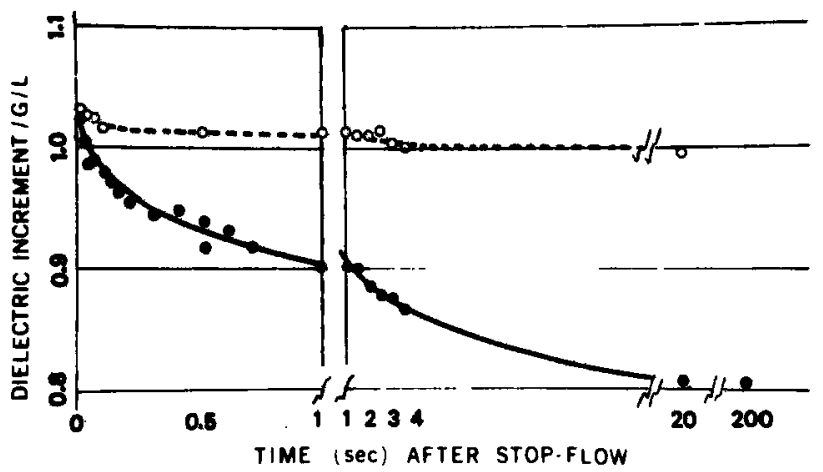

Figure 5. Dielectric Increment as a function of time after mixing in a stop-flow cell (FIGURE 4). (•) = Solution of HSA (LHA76QE1), 66.1 g/1 in water, mixed with solution ImM N-Acetyl-DL-Tryptophan in water, at $0^{\circ} \mathrm{C}, G_{0}=6.2 \mu \mathrm{mho} / \mathrm{cm} ;(0)=$ Control, same HSA solution mixed with water, $G_{0}=5.1 \mu \mathrm{mho} / \mathrm{cm}$.

in terms of a reaction assumed to be separable from the longer-time event. As such, using the initial (high) $C_{\text {tot }}$ and the $1 \mathrm{sec}$ (low) value of $C_{\text {tot }}$ as parameters reflecting an unreacted and a reacted state for the HSA molecule, we obtain in FigURE 6 a graphical representation of the extent of reaction as a function of time, indicating a good fit to first-order kinetics with a rate constant of $(3 \pm 1 / 2) \mathrm{sec}^{-1}$.

This result, unexpected to be sure, becomes understandable in the light of the other information from the same sequence of pulse responses. The value of $G_{0}$, not presented graphically because it would be a straight horizontal line, is virtually

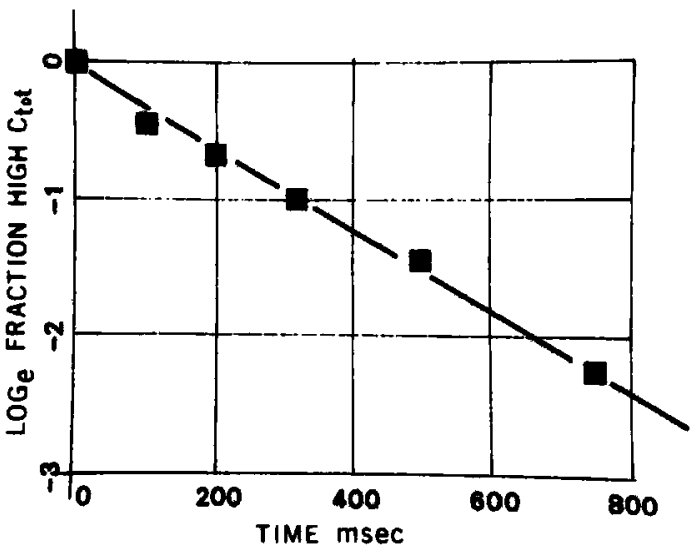

FIgURE 6. Data of Figure 5 from $t=0$ to $t=1 \mathrm{sec}$, displayed as a fraction of protein remaining in the high dielectric increment state. For this calculation, the assumption was made that the decline in dielectric increment after $I \mathrm{sec}$ is a separable decay. 
invariant with time after the first data point, having a value only slightly different from that for the control experiment, and far lower than the total conductance of the unreacted mixture that can be estimated from calculations based on the conductance of free ligand. This is a relatively complicated calculation, involving the degree of ionization of the ligand and the extent of $\mathrm{pH}$ buffering by the protein, but the conclusion is clear, that by the time of the first measurement virtually all free ligand has been immobilized, suggesting that it has been bound by the protein, but not yet in the configuration that it reaches at equilibrium.

The inference from the conductivity data is that the time constant of the initial binding step is less than $1 \mathrm{msec}$, giving a second-order rate constant $k>$ $10^{6} \mathrm{M}^{-1} \mathrm{sec}^{-1}$. This suggests a diffusion-limited first step. With ligand and protein already in place, it is to be expected that the subsequent step be of first-order kinetics, as indeed the data suggest.

These experiments have also been done with oleic acid as ligand, and the same general kinetics are evident.

\section{Conclusion}

The pulse-response technique described has demonstrated that the binding of $N$-acetyl tryptophan and oleic acid by HSA takes place in at least two steps, the first occurring quite rapidly, producing a complex that removes the ligand from the free state but does not involve the characteristic change in dipole moment associated with the final bound state. Presumably, the ligand has found its albumin molecule and attached itself to one of the more accessible low-affinity sites.

Following such an attachment is a second, much slower step, with first-order kinetics, during which the ligand and the protein rearrange their configuration, perhaps involving an opening and reclosing of the $N$ configuration, after which the ligand has been bound at the more interior, high-affinity site.

These conclusions are very much consistent with all that equilibrium studies have revealed about the binding of fatty acids to HSA. They are indeed a confirmation of the view of two distinct types of binding sites.

It should be noted that the experiments on the albumin-fatty acid system done thus far are quite limited, and still allow for various interpretations.

Many obvious variations on the experiments described can now be done, and may help develop a better understanding of the functioning of the primary organic binding site or sites on HSA.

The pulse-response method has proved its feasability and its usefulness as a probe of chemical processes. It is our hope that it will also prove versatile enough to access other systems to dielectric measurement, systems too fragile or too changeable to hold still for steady-state measurements.

\section{ACKNOWLEDGMENTS}

This work would not have been possible without the generous cooperation of Dr. John Mercer at the Blood Fractionation Laboratory, Michigan Department of Public Health, Lansing, Mich. who supplied us with high quality HSA. 


\section{REFERENCES}

1. Scheider, W., H. M. Dintzis \& J. L. Oncley. 1976. Changes in the electric dipole vector of human serum albumin due to complexing with fatty acids. Biophys. J. 16: 417 .

2. Debye, P. 1929. Polar Molecules. Chem. Cat. Co. New York, N.Y. Reprinted by Dover Publishing Co. New York, N.Y.

3. Wyman, J., JR. 1936. Polarization and dielectric constant of liquids. J. Am. Chem. Soc. 58: 1482.

4. ONCLEY, J. L. 1942. The investigation of proteins by dielectric measurements. Chem. Rev. 30: 433 .

5. Husimi, Y. \& A. WaDA. 1976. Time-domain measurement of dielectric dispersion as a response to pseudorandom noise. Rev. Sci. Instrum. 47: 213.

6. Campbell, G. A. \& R. M. Foster. 1948. Fourier Integrals. D. Van Nostrand Co. Princeton, N.J.

7. COLE, K. S. \& R. H. Cole. 1941. Dispersion and absorption in dielectrics. J. Chem. Phys. 9: 341 .

8. COOLEY, J. W. \& J. W. TUKEY. 1965. An algorithm for the machine calculation of complex Fourier series. Math. Comp. 19: 297

9. Moser, P., P. G. Squire \& C. T. O'Konski. 1966. Electric polarization in proteinsdielectric dispersion and Kerr effect studies of Isoionic bovine serum albumin. J. Phys. Chem. 70: 744 .

10. Squire, P. G., P. Moser \& C. T. O'Konski. 1968. The hydrodynamic properties of bovine serum albumin monomer and dimer. Biochemistry 7:4261.

11. Soetewey, F., M. Rosseneu-Motreff, R. Lamote \& H. Peeters. 1972. Size and shape determination of native and defatted bovine serum albumin monomers. J. Biochem. 71: 705.

12. Goodman, D. S. 1958. The interaction of human serum albumin with long-chain fatty acid anions. J. Am. Chem. Soc. 80: 3892 .

13. Spector, A. A., K. John \& J. E. Fletcher. 1969. Binding of long-chain fatty acids to bovine serum albumin. J. Lipid Res. 10: 56.

14. Goodman, D. S. 1957. Preparation of human serum albumin free of long-chain fatty acids. Science 125: 1296.

15. CHEN, R. F. 1967. Removal of fatty acids from serum albumin by charcoal treatment. J. Biol. Chem. 242: 173.

16. Odell, G. B. 1973. Influence of binding on the toxicity of bilirubin, Ann. N.Y. Acad. Sci. 226: 225.

17. KING, T. P. 1973. Location of the primary organic ligand binding site of bovine plasma albumin. Ann. N.Y. Acad. Sci. 226: 94.

18. BRown, J. R. 1974. Structure of serum albumin: disulfide bridges. Fed. Proc. 33: 1389.

19. Chance, B. 1976. Rapid flow methods. Tech. Chem. (N.Y.) 6 (Part 2): 5.

20. Cohn, E. J., J. L. Oncley, L. E. Strong, W. L. Hughes, Jr. \& S. H. Armstrong, JR. 1944. The characterization of the protein fractions of human plasma. J. Clin. Inv. 23:417.

21. Scheider, W. \& J. K. Fuller. 1970. An effective method for defatting albumin using resin columns. Biochim. Biophys. Acta $221: 376$.

22. Dintzis, H. M. 1952. Dielectric properties of human mercaptalbumin. Ph.D. Thesis. Harvard Univ. Cambridge, Mass. 